\title{
EXISTENCE RESULTS FOR QUASISTATIC CONTACT PROBLEM WITH INTERNAL STATE VARIABLES
}

\author{
N. Lebri \\ Department of Mathematics \\ Faculty of Sciences \\ University of Farhat Abbas \\ Setif, 19000, ALGERIA
}

\begin{abstract}
The subject of this work is the study of a value problem describing the quasistatic evolution of semilinear retetype viscoplastic models with internal state variables, and we suppose the problem of Tresca's Friction law at the presence of recal forces. The existence and uniqueness of the solution is proved using results of evolutionary variational inequalities and a fixed point theorem.
\end{abstract}

AMS Subject Classification: 74M15, 74S05, 65M60

Key Words: viscoplasticity, Trasca's law, variational inequality, fixed point

\section{Introduction}

For the bilateral contact problem studied in this paper, the friction is modelled by tresca's law with internal state variables.

In Section 2 we present some basic notations on the functional spaces and some hypothesis used in the following.

The model is described in Section 3, where the variational formulation is given. In Section 4, using results of evolutionary variational inequalities and a fixed point theorem, an existence and uniqueness result is obtained. Finally,

Received: January 19, 2013

(c) 2013 Academic Publications, Ltd. url: www.acadpubl.eu 
in Section 5, we establish a study of the dependence of the solution by the parameter.

\section{Statement of the Problem. Hypothesis}

We assume the contact is bilateral, i.e. there is no loss of the contact during the process. Thus, the normal displacement $u_{\nu}$ vanishes on $\Gamma_{3}$ at any time. We model the friction with the tresca friction law.

Let $\Omega \subset \mathbb{R}^{N}(N=1,2,3)$ a bounded domain and whose boundary $\Gamma$ assumed to be sufficiently smooth, is partitioned into three disjoint measurable parts $\Gamma_{1}, \Gamma_{2}$ and $\Gamma_{3}$. Let meas $\Gamma_{1}>0$, let $T>0$ be a time.

Let $M$ be a natural number. We consider the following mixed problem

$$
\begin{gathered}
\dot{\sigma}=\xi(\varepsilon(\dot{u}))+G(\sigma, \varepsilon(u), k, \theta) \text { in } \Omega \times[0, T] . \\
\dot{k}=\phi(\varepsilon(u), \sigma, k, \theta) \text { in } \Omega \times[0, T] . \\
D i v \sigma+f_{0}=0 \text { in } \Omega \times[0, T] . \\
\left.\begin{array}{c}
u=0 \text { on } \Gamma_{1} \times[0, T] . \\
\sigma \nu+\Phi(x) u=f_{2} \text { on } \Gamma_{2} \times[0, T] . \\
u_{\nu}=0,\left\|\sigma_{\tau}\right\| \leq g, \\
\left\|\sigma_{\tau}\right\|<g \Rightarrow \dot{u}_{\tau}=0, \\
\left\|\sigma_{\tau}\right\|=g \Rightarrow \exists \lambda>\sigma_{\tau}=-\lambda \dot{u}_{\tau}
\end{array}\right\} \text { on } \Gamma_{3} \times[0, T] . \\
u(0)=u_{0}, \sigma(0)=\sigma_{0}, k(0)=k_{0} \text { in } \Omega .
\end{gathered}
$$

Here the unknowns are the functions $u: \Omega \times(0, T) \rightarrow \mathbb{R}^{N}, \sigma: \Omega \times(0, T) \rightarrow S_{N}$ and $k: \Omega \times(0, T) \rightarrow \mathbb{R}^{M}$ in which $k$ may be interpreted as an internal state variable and $\xi, k$ and $G$ are given constitutive functions. $\Phi$ represent rappel force.

In (1)-(7) $u$ represents the displacement function, $\sigma$ represents the stress function, $\varepsilon(u)$ denotes the small strain tensor, $f_{0}$ is given body force, $f_{2}$ is given boundary data, and $u_{0}, \sigma_{0}$ and $k_{0}$ are the initial data. In (6), $g \geqslant 0$ is the friction bound function, i.e. magnitude of the limiting friction traction at which slip begins. The strict inequality in (6) holds in the stick zone and the equality holds in the slip zone. The equation (5) means that the Cauchy vector $\sigma \nu$ is proportional on the displacement. 
Viscoplastic models of the form (1), (2) are used in order to model the behavior of real bodies for which the plastic rate of deformation depends also on an internal state variable.

Some of the internal state variables considered by many authors are the plastic strain, a number of tensor variables that takes into account the spatial display of dislocation for the internal state variables. Here we suppose that $k$ is a vector-valued function which satisfies (2) where $\phi$ is a given function.

In order to obtain variational formulations for the problem (1)-(7), let us consider the following notations

$$
\begin{aligned}
& H=\left\{v=\left(v_{i}\right) / v_{i} \in \mathbb{L}^{2}(\Omega), i=\overline{1, N}\right\} \\
& H_{1}=\left\{v=\left(v_{i}\right) / v_{i} \in H^{1}(\Omega), i=\overline{1, N}\right\}, \\
& V=\left\{v \in H_{1}: v=0 \text { a.e on } \Gamma_{1}\right\} \\
& V 1=\left\{v \in V / v_{\nu}=0 \text { a.e on } \Gamma_{3}\right\}, \\
& Q=\left\{\tau=\left(\tau_{i j}\right):\left(\tau_{i j}\right)=\left(\tau_{j i}\right) \in \mathbb{L}^{2}(\Omega), 1 \leq i, j \leq p\right\}, \\
& Q_{1}=\{\tau \in Q, \quad \text { Div } \tau \in H\} .
\end{aligned}
$$

The spaces $H, H_{1}, V, V_{1}, Q, Q_{1}$ are real Hilbert spaces endowed with the canonical inner products denoted by $\langle., .\rangle_{H},\langle., .\rangle_{H_{1}},\langle., .\rangle_{V},\langle., .\rangle_{V_{1}},\langle., .\rangle_{Q}$, $\langle., .\rangle_{Q_{1}}$ respectively.

We assume that the force and traction satisfy

$$
f_{0} \in W^{1, \infty}(0, T, H), f_{2} \in W^{1, \infty}\left(0, T, H_{\Gamma_{2}}\right),
$$

and the friction bound satisfies

$$
g \in L^{\infty}\left(\Gamma_{3}\right), g \geqslant 0 \text { on } \Gamma_{3}, \theta \in L^{\infty}\left(0, T, L^{2}(\Omega)^{M}\right) .
$$

Denote by $f(t)$ the element of $V_{1}$ giving by

$$
\langle f(t), v\rangle_{V}=\int_{\Omega} f_{0} \cdot v d s+\int_{\Gamma_{2}} f_{2} \cdot v d a \quad \forall v \in V_{1} .
$$

For all $t \in[0, T]$ let $j: V_{1} \rightarrow \mathbb{R}_{+}$be the functional

$$
j(v)=\int_{\Gamma_{2}} g\left\|v_{\tau}\right\| d a .
$$

For all $t \in[0, T]$, we note that conditions (8) and (9) imply

$$
f \in W^{1, \infty}\left(0, T, V_{1}\right)
$$

and

$$
j(v) \leq c\|g\|_{\mathbb{L}^{\infty}\left(\Gamma_{3}\right)}\|v\| \quad \forall v \in V_{1}
$$


We also introduce the notation $\Sigma(t)$ defined by

$$
\begin{array}{r}
\Sigma(t)=\left\{\tau \in Q: \quad\langle\tau, \varepsilon(v)\rangle_{Q}+\left\langle\Phi u_{0}, v\right\rangle L^{2}\left(\Gamma_{2}\right)+j(v) \geq\langle f(t), v\rangle_{V}, \forall v \in V_{1}\right\} \\
t \in \overline{[0, T]} .
\end{array}
$$

Finally, assume that the initial data satisfy

$$
u_{0} \in V_{1}, \sigma_{0} \in \Sigma(0)
$$

We have the following result

\section{Variational Formulation}

Lemma 1. If $u$ and $\sigma$ are regular functions satisfying (3)-(6) then for all $t \in[0, T]$,

$$
\begin{aligned}
u(t) \in V_{1}, \quad\langle\sigma(t), \varepsilon(v)- & \varepsilon(\dot{u}(t))\rangle_{Q}+\langle\Phi u(t), v-\dot{u}(t)\rangle_{\mathbb{L}^{2}\left(\Gamma_{2}\right)^{N}} \\
& +j(v)-j(\dot{u}(t)) \geq\langle f(t), v-\dot{u}(t)\rangle_{V} \quad \forall v \in V_{1} .
\end{aligned}
$$

Proof. Let $t \in[0, T]$ and $v \in V_{1}$ be an arbitrary test function. Multiplying the equation $(3)$ by $v-\dot{u}(t)$ and integrating by parts, we obtain

$$
\langle\sigma(t), \varepsilon(v)-\varepsilon(\dot{u}(t))\rangle_{Q}=\int_{\Omega} f_{0}(t) .(v-\dot{u}(t)) d s+\int_{\Gamma} \sigma \nu \cdot(v-\dot{u}(t)) d a .
$$

Using the boundary conditions (4) and (5) we can replace the right band side of the above equality by

$$
\langle f(t), v-\dot{u}(t)\rangle_{V}-\int_{\Gamma_{2}} \Phi u \cdot(v-\dot{u}(t)) d a+\int_{\Gamma_{3}} \sigma \nu \cdot(v-\dot{u}(t)) d a .
$$

From the contact boundary condition (6) we derive the relation

$$
\sigma_{\tau}(t) \cdot \dot{u}_{\tau}(t)=-g\left\|\dot{u}_{\tau}(t)\right\| \quad \text { on } \Gamma_{3} .
$$

Thus, on $\Gamma_{3}$ :

$$
\begin{aligned}
\sigma \nu \cdot(v-\dot{u}(t)) & =\sigma_{\nu}(t) \cdot\left(v_{\nu}-\dot{u}_{v}(t)\right)+\sigma_{\tau}(t) \cdot\left(v_{\tau}-\dot{u}_{\tau}(t)\right) \\
& =\sigma_{\tau}(t) \cdot v_{\tau}-\sigma_{\tau}(t) \cdot \dot{u}_{\tau}(t)
\end{aligned}
$$




$$
\begin{aligned}
& =\sigma_{\tau}(t) \cdot v_{\tau}+g\left\|\dot{u}_{\tau}(t)\right\| \\
& \geq g\left(\left\|\dot{u}_{\tau}(t)\right\|-\left\|v_{\tau}\right\|\right) .
\end{aligned}
$$

Therefore

$$
\int_{\Gamma_{3}} \sigma \nu \cdot(v-\dot{u}(t)) d a \geq j(\dot{u}(t))-j(v),
$$

and we have

$$
\langle\sigma(t), \varepsilon(v)-\varepsilon(\dot{u}(t))\rangle_{Q}+\langle\Phi w, v-\dot{u}(t)\rangle_{\mathbb{L}^{2}\left(\Gamma_{2}\right)^{N}} \geq\langle f(t), v-\dot{u}(t)\rangle_{V}+j(\dot{u}(t)-j(v),
$$

i.e. the inequality (12) holds true.

The previous lemma leads us to consider the following formulations of the frictional problem (1)-(7).

Problem 2. Find a displacement field $u:[0, T] \rightarrow \mathbb{V}_{1}$, a stress field $\sigma:[0, T] \rightarrow Q_{1}$, and $k:[0, T] \rightarrow \mathbb{R}^{M}:$

$$
\begin{gathered}
\dot{\sigma}(t)=\xi(\varepsilon(\dot{u}))+G(\sigma, \varepsilon(u), k, \theta) \text { a.e. } t \in(0, T), \\
\dot{k}=\phi(\varepsilon(u), \sigma, k, \theta), \\
\langle\sigma(t), \varepsilon(v)-\varepsilon(\dot{u}(t))\rangle_{Q}+\langle\Phi u(t), v-\dot{u}(t)\rangle_{\mathbb{L}^{2}\left(\Gamma_{2}\right)^{N}} \\
+j(v)-j(\dot{u}(t)) \geq\langle f(t), v-\dot{u}(t)\rangle_{V} \quad \forall v \in V_{1}, \\
u(0)=u_{0}, \sigma(0)=\sigma_{0}, k(0)=k_{0} .
\end{gathered}
$$

\section{Existence and Uniqueness Results}

In the study of the problem (1)-(7), we consider the following assumptions:

$$
\left\{\begin{array}{l}
\xi: \Omega \times S_{N} \rightarrow S_{N} \\
\text { (a) } \xi_{i j k h} \in L^{\infty}(\Omega) \text { for all } i, j, k, h=\overline{0, N} \\
\text { (b) } \xi \sigma . \tau=\xi . \sigma \tau \forall \sigma, \tau \in S_{N} . \\
\text { (c) } \exists \alpha>0 / \xi \sigma . \sigma \geqslant \alpha|\sigma|^{2} \forall \sigma \in S_{N} .
\end{array}\right.
$$

$\left\{\begin{array}{l}G: \Omega \times S_{N} \times S_{N} \times \mathbb{R}^{M} \times \mathbb{R}^{M} \rightarrow S_{N} \\ (\mathbf{a}) \quad \exists \tilde{L}>0 \text { that } \forall \sigma_{1}, \sigma_{2}, \varepsilon_{1}, \varepsilon_{2} \in \\ \mid G\left(x, \sigma_{1}, \varepsilon_{1}, k_{1}, \theta_{1}\right)-G\left(x, \sigma_{2}, \varepsilon_{2}, k_{2}\right. \\ \leq \tilde{L}\left(\left|\sigma_{1}-\sigma_{2}\right|+\left|\varepsilon_{1}-\varepsilon_{2}\right|+\left|k_{1}-k_{2}\right|\right. \\ \text { (b) } x \rightarrow G(x, \sigma, \varepsilon, k, \theta) \text { is mesurable on } \Omega \\ \text { to the Lebesgue measure on } \\ \text { for all } \sigma, \varepsilon \in S_{N}, k, \theta \in \mathbb{R}^{M} \\ (\mathbf{c}) x \rightarrow G(x, 0,0,0) \in \mathbb{L}^{2}(\Omega)^{N \times N}\end{array}\right.$ 


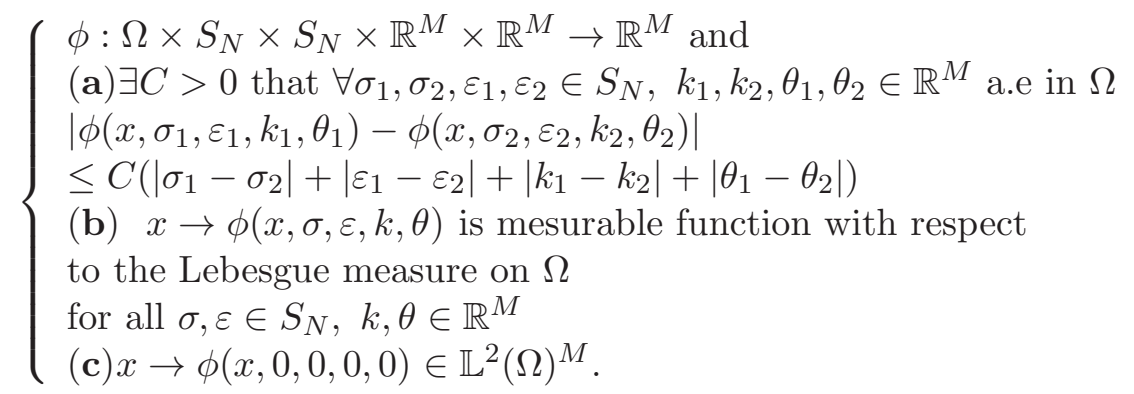

The mapping $\Phi: \Gamma_{2} \rightarrow S_{N}$ is such that:

$$
\begin{aligned}
& \left\{\begin{array}{l}
\Phi(x) u . u \geq 0 \text { on } \Gamma_{2} . \\
\Phi_{k m} \in L^{\infty}\left(\bar{\Gamma}_{2}\right), \quad \Phi_{k m}=\Phi_{m k} \text { for all } k, m=1, \ldots, N
\end{array}\right. \\
& k_{0} \in \mathbb{L}^{2}(\Omega)^{M} .
\end{aligned}
$$

The main result in this section is:

Theorem 1. Assume (8), (9), (14), (19) and (20)-(23). Then the problem (2) has a unique solution

$$
u \in W^{1, \infty}\left(0, T, V_{1}\right), \sigma \in W^{1, \infty}\left(0, T, Q_{1}\right), k \in W^{1, \infty}\left(0, T, \mathbb{L}^{2}(\Omega)^{M}\right) .
$$

Proof. We start by the existence part.

Let $X$ be the product Hilbert space $X=Q \times \mathbb{L}^{2}(\Omega)^{M}$ and let $\eta=\left(\eta^{1}, \eta^{2}\right)$ in $C^{0}(0, T, X)$.

We define the function $Z_{\eta}=Z_{\eta}^{1}+Z_{\eta}^{2} \in C^{0}(0, T, X)$ by

$$
Z_{\eta}(t)=\int_{0}^{t} \eta(s) d s+Z_{0}
$$

where

$$
Z_{0}=\left(\sigma_{0}-\xi \varepsilon\left(u_{0}\right), k_{0}\right)
$$

Consider the following auxiliary variational problem.

Problem 2. Find a displacement field $u_{\eta}:[0, T] \rightarrow \mathbb{V}_{1}$, a stress field $\sigma_{\eta}:[0, T] \rightarrow Q_{1}$, and $k \in W^{1, \infty}\left(0, T, \mathbb{L}^{2}(\Omega)^{M}\right):$

$$
\begin{gathered}
\sigma_{\eta}(t)=\xi\left(\varepsilon\left(u_{\eta}\right)\right)+Z_{\eta}^{1}(t) \text { in } \Omega \times(0, T), \\
\left\langle\sigma_{\eta}(t), \varepsilon(v)-\varepsilon\left(\dot{u}_{\eta}(t)\right)\right\rangle_{Q}+\langle\Phi u(t), v-\dot{u}(t)\rangle_{\mathbb{L}^{2}\left(\Gamma_{2}\right)^{N}}+j(v)-j\left(\dot{u}_{\eta}(t)\right) \\
\geq\left\langle f(t), v-\dot{u}_{\eta}(t)\right\rangle_{V} \forall v \in V_{1},
\end{gathered}
$$




$$
u_{\eta}(0)=u_{0}, \sigma_{\eta}(0)=\sigma_{0}, k(0)=k_{0} .
$$

Lemma 2. The variational problem (2) has a unique solution $u_{\eta} \in W^{1, \infty}(0$, $\left.T, V_{1}\right), \sigma_{\eta} \in W^{1, \infty}\left(0, T, Q_{1}\right)$.

Proof. Define a bilinear form $a: V_{1} \times V_{1} \rightarrow \mathbb{R}$ by

$$
a(u, v)=\langle\xi(\varepsilon(u)), \varepsilon(v)\rangle_{Q}+\langle\Phi u(t), v-\dot{u}(t)\rangle_{\mathbb{L}^{2}\left(\Gamma_{2}\right)^{N}} \quad \forall u, v \in V_{1} .
$$

It follows from the assumptions (19) on $\xi$ that $a(u, v)$ is continuous, symmetric and $V_{1}$-elliptic. Also by (9) we see $j$ is a continuous seminorm on $V_{1}$.

Let $f_{\eta}:[0, T] \rightarrow V_{1}$ defined by

$$
\left\langle f_{\eta}, v\right\rangle_{V}=\langle f(t), v\rangle_{V}-\left\langle Z_{\eta}^{1}(t), \varepsilon(v)\right\rangle_{Q} \quad \forall v \in V_{1} .
$$

Since $f \in W^{1, \infty}\left([0, T], V_{1}\right)$ and $Z_{\eta}^{1} \in W^{1, \infty}([0, T], Q)$, we have $f_{\eta} \in$ $W^{1, \infty}\left([0, T], V_{1}\right)$.

Moreover, since $\sigma_{0}=\xi \varepsilon\left(u_{0}\right)+Z_{0}^{1}=\xi \varepsilon\left(u_{0}\right)+Z_{\eta}^{1}(0)$ by (26) and (25), the assumption $\sigma_{0} \in \Sigma(0)$ from (14) can be written as

$$
\left\langle\xi \varepsilon\left(u_{0}\right), \varepsilon(v)\right\rangle_{Q}+\left\langle Z_{\eta}^{1}(0), \varepsilon(v)\right\rangle_{Q}+j(v) \geq\langle f(0), v\rangle_{V} \quad \forall v \in V_{1} .
$$

Applying a theorem in ([3] page70) we see that there exists a unique solution $u_{\eta} \in W^{1, \infty}\left(0, T, V_{1}\right)$ to the problem

$$
\begin{aligned}
a\left(u_{\eta}(t), v-\dot{u}_{\eta}(t)\right)+j(v)-j\left(\dot{u}_{\eta}(t)\right) & \geq\left\langle f_{\eta}(t), v-\dot{u}_{\eta}(t)\right\rangle_{V} \forall v \in V_{1}, \\
u_{\eta}(0) & =u_{0} .
\end{aligned}
$$

Let $\sigma_{\eta} \in W^{1, \infty}(0, T, Q)$ be given by $(26)$. Then $\left(u_{\eta}, \sigma_{\eta}\right)$ is a solution for (27)-(29). From inequality (28) we obtain $D i v \sigma_{\eta}+f_{0}=0$ a. e in $\Omega \times(0, T)$. Therefore, $\sigma_{\eta} \in W^{1, \infty}\left(0, T, Q_{1}\right)$.

Let $k_{\eta} \in L^{\infty}\left(0, T, \mathbb{L}^{2}(\Omega)^{M}\right)$ be the function defined by

$$
k_{\eta}=Z_{\eta}^{2}
$$

Finally, the uniqueness part of the lemma follows from the unique solvability of the variational inequality problem (30)-(31).

We consider next an operator $\Lambda: L^{\infty}(0, T, X) \rightarrow L^{\infty}(0, T, X)$ defined by

$$
\begin{aligned}
\Lambda \eta(t)=\left(G\left(\sigma_{\eta}(t), \varepsilon\left(u_{\eta}\right), k_{\eta}(t), \theta\right), \phi\left(\sigma_{\eta}(t), \varepsilon\left(u_{\eta}\right),\right.\right. & \left.\left.k_{\eta}(t), \theta\right)\right) \\
& \text { for all } t \in[0, T] .
\end{aligned}
$$


Here $\left(u_{\eta}, \sigma_{\eta}, k_{\eta}\right)$ is solution of problem (3).

Lemma 3. The operator $\Lambda$ has a unique fixed point $\eta_{*} \in L^{\infty}(0, T, X)$.

Proof. Let $\eta_{1}, \eta_{2} \in L^{\infty}(0, T, X)$ and denote $u_{i}=u_{\eta i_{i}}, \sigma_{i_{i}}=\sigma_{\eta i_{i}}, Z_{i_{i}}=Z_{\eta i_{i}}$, $Z_{i}=Z_{i}^{1}+Z_{i}^{2}$ and $\eta_{i}=\left(\eta_{i}^{1}, \eta_{i}^{2}\right)$ for all $i=1,2$. Rewrite (27) and (28) for $u_{1}$ and $u_{2}$ as

$a\left(u_{1}(t), v-\dot{u}_{1}(t)\right)+\left\langle Z_{1}^{1}, \varepsilon(v)-\varepsilon\left(\dot{u}_{1}(t)\right)\right\rangle_{Q}+j(v)-j\left(\dot{u}_{1}(t)\right) \geq\left\langle f(t), v-\dot{u}_{1}(t)\right\rangle_{V}$,

$a\left(u_{2}(t), v-\dot{u}_{2}(t)\right)+\left\langle Z_{2}^{1}, \varepsilon(v)-\varepsilon\left(\dot{u}_{2}(t)\right)\right\rangle_{Q}+j(v)-j\left(\dot{u}_{2}(t)\right) \geq\left\langle f(t), v-\dot{u}_{2}(t)\right\rangle_{V}$.

For all $v \in V_{1}$, a. e on $(0, T)$. we take $v=\dot{u}_{1}$ in the first inequality, $v=\dot{u}_{2}$ in the second inequality, and add the two inequalities to obtain

$$
a\left(u_{2}-u_{1}, \dot{u}_{2}-\dot{u}_{1}\right) \leq-\left\langle Z_{1}^{1}-Z_{2}^{1}, \varepsilon\left(\dot{u}_{1}(t)\right)-\varepsilon\left(\dot{u}_{2}(t)\right)\right\rangle_{Q} \text { a.e. on }(0, T) .
$$

Let $t \in \overline{[0, T]}$. Integrate the inequality from 0 to $t$

$$
\begin{aligned}
\frac{1}{2} \frac{d}{d t} a\left(u_{2}-u_{1}, u_{2}-u_{1}\right) \leq- & \left\langle Z_{1}^{1}-Z_{2}^{1}, \varepsilon\left(u_{1}(t)\right)-\varepsilon\left(u_{2}(t)\right)\right\rangle_{Q} \\
& +\int_{0}^{t}\left\langle\eta_{1}^{1}(s)-\eta_{2}^{1}(s), \varepsilon\left(u_{1}(s)\right)-\varepsilon\left(u_{2}(s)\right)\right\rangle_{Q} d s
\end{aligned}
$$

Then

$$
\begin{aligned}
c\left\|u_{1}(t)-u_{2}(t)\right\|_{V}^{2} \leq \| Z_{1}^{1}(t)- & Z_{2}^{1}(t)\left\|_{Q}\right\| u_{1}(t)-u_{2}(t) \|_{V} \\
& +\int_{0}^{t}\left\|\eta_{1}^{1}(s)-\eta_{2}^{1}(s)\right\|_{Q}\left\|u_{1}(s)-u_{2}(s)\right\|_{V} d s .
\end{aligned}
$$

Now

$$
Z_{1}(t)-Z_{2}(t)=\int_{0}^{t}\left(\eta_{1}^{1}(s)-\eta_{2}^{1}(s)\right) d s
$$

So

$$
\left\|Z_{1}^{1}(t)-Z_{2}^{1}(t)\right\|_{Q} \leq \int_{0}^{t}\left\|\eta_{1}^{1}(s)-\eta_{2}^{1}(s)\right\|_{Q} d s .
$$

Therefore, we have

$$
\left\|u_{1}(t)-u_{2}(t)\right\|_{V}^{2} \leq c \int_{0}^{t}\left\|\eta_{1}^{1}(s)-\eta_{2}^{1}(s)\right\|_{Q}^{2} d s+\int_{0}^{t}\left\|u_{1}(s)-u_{2}(s)\right\|_{V}^{2} d s .
$$


Applying the Gronwal inequality, we obtain

$$
\left\|u_{1}(t)-u_{2}(t)\right\|_{V}^{2} \leq c \int_{0}^{t}\left\|\eta_{1}^{1}(s)-\eta_{2}^{1}(s)\right\|_{Q}^{2} d s .
$$

By the definition (27) and (33) we have

$$
\left\|\sigma_{1}(t)-\sigma_{2}(t)\right\|_{Q}^{2} \leq c \int_{0}^{t}\left\|\eta_{1}^{1}(s)-\eta_{2}^{1}(s)\right\|_{Q}^{2} d s .
$$

Using (32) we have

$$
\begin{aligned}
\left\|k_{1}-k_{2}\right\|_{\mathbb{L}^{2}(\Omega)^{M}}=\left\|Z_{1}^{2}-Z_{2}^{2}\right\|_{\mathbb{L}^{2}(\Omega)^{M}} \leq\left\|Z_{1}-Z_{2}\right\|_{\mathbb{L}^{2}(\Omega)^{M}} & \\
& \leq \int_{0}^{t}\left\|\eta_{1}^{1}(s)-\eta_{2}^{1}(s)\right\|_{Q}^{2} d s .
\end{aligned}
$$

Finally,

$$
\begin{aligned}
\Lambda \eta_{1}(t)-\Lambda \eta_{2}(t)= & \left(G\left(\sigma_{\eta_{1}}(t), \varepsilon\left(u_{\eta_{1}}\right), K_{\eta_{1}}(t), \theta\right), \phi\left(\sigma_{\eta_{1}}(t), \varepsilon\left(u_{\eta_{1}}\right), K_{\eta_{1}}(t), \theta\right)\right) \\
& -\left(G\left(\sigma_{\eta_{2}}(t), \varepsilon\left(u_{\eta_{2}}\right), K_{\eta_{2}}(t), \theta\right), \phi\left(\sigma_{\eta 2}(t), \varepsilon\left(u_{\eta_{2}}\right), K_{\eta_{2}}(t), \theta\right)\right)
\end{aligned}
$$

Using now (20), (21), (34) and (33) we get

$$
\left|\Lambda \eta_{1}(t)-\Lambda \eta_{2}(t)\right|_{X} \leq \tilde{C} \int_{0}^{t}\left|\eta_{1}(s)-\eta_{2}(s)\right|_{X} d s \text { for all } t \in(0, T) .
$$

Lemma (3) follows from (36) and an application of Banach's fixed point theorem.

Now we are ready to prove Theorem (1).

\section{Proof of Theorem 1}

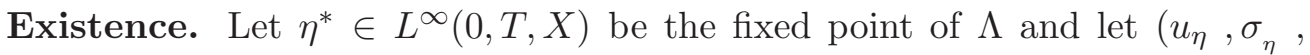
$K_{\eta^{*}}$ ) be the solution of problem (3) for $\eta=\eta^{*}$. Using (27) and (25) we have

$$
\dot{\sigma}_{\eta^{*}}(t)=\xi\left(\varepsilon\left(\dot{u}_{\eta^{*}}(t)\right)\right)+\eta^{*}(t) \text { a.e. } t \in(0, T) .
$$

Since

$$
\eta^{*}(t)=\Lambda \eta^{*}(t)=\left(G\left(\sigma_{\eta}(t), \varepsilon\left(u_{\eta}\right), K_{\eta}(t), \theta\right), \phi\left(\sigma_{\eta}(t), \varepsilon\left(u_{\eta}\right), K_{\eta}(t), \theta\right)\right)
$$


it follows that $\left(u_{\eta^{*}}, \sigma_{\eta^{*}}, K_{\eta^{*}}\right)$ satisfies (16). Using now (28) and (29) we conclude that is a solution of problem (2) with regularity (24).

Uniqueness. The uniqueness part follows from the uniqueness of the fixed point of the operator $\Lambda$.

\section{The Dependence of the Solution on the Given Parameter}

Theorem 2. Under the assumptions, let $\left(u_{i}, \sigma_{i}\right),(i=1,2)$ be the variational solution of the Problem 2 associated to parameter $\theta_{i}$ such that:

Under the assumptions (19)-(21), let $\left(u_{i}, \sigma_{i}\right)(i=1,2)$ be the variational solution of the problem 2 associated to the parameter $\theta_{i} \in \mathbb{L}^{2}(\Omega)^{M}$ is satisfied. Then there exists a positive constant $C>0$ depending to $\Omega, \Gamma_{1}$ and $\Gamma$ such that

$$
\left|u_{1}-u_{2}\right|_{V}+\left|\sigma_{1}-\sigma_{2}\right|_{Q}+\left|k_{1}-k_{2}\right|_{\mathbb{L}^{2}(\Omega)^{M}} \leq C\left|\theta_{1}-\theta_{2}\right|_{\mathbb{L}^{2}(\Omega)^{M}} .
$$

Proof. Let $\left(u_{i}, \sigma_{i}\right),(i=1,2)$, be a variational solution of the Problem 2 then

$$
\begin{aligned}
\left\langle\sigma_{i}(t), \varepsilon(v)-\varepsilon(\dot{u}(t))\right\rangle_{Q} & +\left\langle\Phi u_{i}(t), v-\dot{u}_{i}(t)\right\rangle_{\mathbb{L}^{2}\left(\Gamma_{2}\right)^{N}} \\
& +j(v)-j\left(\dot{u}_{i}(t)\right) \geq\left\langle f(t), v-\dot{u}_{i}(t)\right\rangle_{V} \forall v \in V_{1} .
\end{aligned}
$$

where

$$
\begin{gathered}
\sigma_{i}(t)=\xi\left(\varepsilon\left(u_{i}\right)\right)+Z_{i}(t) \text { a.e. } t \in(0, T) \\
Z_{i}(t)=\int_{0}^{t} G\left(\sigma_{i}(s), \varepsilon\left(u_{i}(s)\right), \theta_{i}(s)\right) d s+\sigma_{0}-\xi \varepsilon\left(u_{0}\right) .
\end{gathered}
$$

Rewriting (37) and (38) for $u_{1}$ and $u_{2}$, we obtain:

$$
\begin{aligned}
& a\left(u_{1}, v-\dot{u}_{1}\right)+\left\langle Z_{1}(t), \varepsilon(v)-\varepsilon\left(\dot{u}_{1}(t)\right)\right\rangle_{Q}+j(v)-j\left(\dot{u}_{1}(t)\right) \geq\left\langle f(t), v-\dot{u}_{1}(t)\right\rangle_{V}, \\
& a\left(u_{2}, v-\dot{u}_{2}\right)+\left\langle Z_{2}(t), \varepsilon(v)-\varepsilon\left(\dot{u}_{2}(t)\right)\right\rangle_{Q}+j(v)-j\left(\dot{u}_{2}(t)\right) \geq\left\langle f(t), v-\dot{u}_{2}(t)\right\rangle_{V} .
\end{aligned}
$$

For all $v \in V_{1}$, a. e on $(0, T)$, we take $v=\dot{u}_{1}$ in the first inequality, $v=\dot{u}_{2}$ in the second inequality, and add the two inequalities to obtain

$$
\frac{1}{2} \frac{d}{d t} a\left(u_{1}-u_{2}, u_{1}-u_{2}\right) \leq-\left\langle Z_{1}-Z_{2}, \varepsilon\left(\dot{u}_{1}(t)\right)-\varepsilon\left(\dot{u}_{2}(t)\right)\right\rangle_{Q} \text { a.e. on }(0, T) \text {. }
$$


Let $t \in \overline{[0, T]}$. Integrate the inequality from 0 to $t$

$$
\begin{aligned}
\frac{1}{2} a\left(u_{2}-u_{1}, u_{2}-u_{1}\right) \leq-\left\langle Z_{1}\right. & \left.-Z_{2}, \varepsilon\left(u_{1}(t)\right)-\varepsilon\left(u_{2}(t)\right)\right\rangle_{Q} \\
& +\int_{0}^{t}\left\langle\eta_{1}(s)-\eta_{2}(s), \varepsilon\left(u_{1}(s)\right)-\varepsilon\left(u_{2}(s)\right)\right\rangle_{Q} d s .
\end{aligned}
$$

Then

$$
\begin{aligned}
& c\left\|u_{1}(t)-u_{2}(t)\right\|_{V}^{2} \leq\left\|Z_{1}(t)-Z_{2}(t)\right\|_{Q}\left\|u_{1}(t)-u_{2}(t)\right\|_{V} \\
+ & \int_{0}^{t}\left\|G\left(\sigma_{1}(s), \varepsilon\left(u_{1}(s)\right), \theta_{1}(s)\right)-G\left(\sigma_{2}(s), \varepsilon\left(u_{2}(s)\right), \theta_{2}(s)\right)\right\|_{Q}\left\|u_{1}(s)-u_{2}(s)\right\|_{V} d s .
\end{aligned}
$$

Now

$$
Z_{1}(t)-Z_{2}(t)=\int_{0}^{t} G\left(\sigma_{1}(s), \varepsilon\left(u_{1}(s)\right), \theta_{1}(s)\right)-G\left(\sigma_{2}(s), \varepsilon\left(u_{2}(s), \theta_{2}(s)\right) d s .\right.
$$

So

$\left\|Z_{1}(t)-Z_{2}(t)\right\|_{Q} \leq \int_{0}^{t} \| G\left(\sigma_{1}(s), \varepsilon\left(u_{1}(s)\right), \theta_{1}(s)\right)-G\left(\sigma_{2}(s), \varepsilon\left(u_{2}(s), \theta_{2}(s)\right) \|_{Q} d s\right.$.

Therefore, we have

$$
\begin{aligned}
\| u_{1}(t) & -u_{2}(t) \|_{V}^{2} \\
\leq c \int_{0}^{t} \| G\left(\sigma_{1}(s), \varepsilon\left(u_{1}(s)\right), \theta_{1}(s)\right)-G\left(\sigma_{2}(s), \varepsilon\left(u_{2}(s), \theta_{2}(s)\right) \|_{Q}^{2} d s\right. & +\int_{0}^{t}\left\|u_{1}(s)-u_{2}(s)\right\|_{V}^{2} d s .
\end{aligned}
$$

Applying the Gronwal inequality, we obtain

$$
\begin{aligned}
\| u_{1}(t) & -u_{2}(t) \|_{V}^{2} \\
& \leq c \int_{0}^{t} \| G\left(\sigma_{1}(s), \varepsilon\left(u_{1}(s)\right), \theta_{1}(s)\right)-G\left(\sigma_{2}(s), \varepsilon\left(u_{2}(s), \theta_{2}(s)\right) \|_{Q}^{2} d s .\right.
\end{aligned}
$$

By the definition (36) and (37) we have

$$
\begin{aligned}
\| \sigma_{1}(t) & -\sigma_{2}(t) \|_{Q}^{2} \\
& \leq c \int_{0}^{t} \| G\left(\sigma_{1}(s), \varepsilon\left(u_{1}(s)\right), \theta_{1}(s)\right)-G\left(\sigma_{2}(s), \varepsilon\left(u_{2}(s)\right), \theta_{2}(s) \|_{Q}^{2} d s .\right.
\end{aligned}
$$


Finally, Using (20), and applying the Gronwal inequality, we obtain

$$
\left|u_{1}-u_{2}\right|_{V}+\left|\sigma_{1}-\sigma_{2}\right|_{Q}+\left|k_{1}-k_{2}\right|_{\mathbb{L}^{2}(\Omega)^{M}} \leq C\left|\theta_{1}-\theta_{2}\right|_{\mathbb{L}^{2}(\Omega)^{M}} .
$$

This theorem proves the dependence of the solution to the parameter $\theta$ and this result is very important from the mechanical point of view because it prove that small perturbations on the parameter $\theta$ gives small perturbations on the solution $(u, \sigma)$ of the mechanical problem $(1)-(7)$.

\section{References}

[1] A. Bassam, Analyse variationnelle de quelques problèmes viscoélastiques et viscoplastiques avec frottement. Thèse de Doctorat d'Etat, Université de Perpignan (2001).

[2] S. Boutechebak \& N. Lebri, Frictionless contact problem non linear elasticity. Anal. Oradea. Fasc. Math. Tom XV (2008). 129-140

[3] H. Brezis, Analyse fonctionnelle. Théorie et applications. Maison. Paris (1987).

[4] O. Chau, Analyse variationnelle et numérique de quelques problèmes aux limites en mécanique du contact. Ph.D. Thesis, University of Perpignan, France (2000).

[5] S. Djabi \& M. Sofonea, A monotony method in quasi-static material with internal state variables. Univ Blaise Pascal (Clermont II ) Série math. (1989).

[6] S. Drabla, Analyse variationnelle de quelques problèmes aux limites en élasticité et en viscoplasticité. Ph.D. Thesis, University of Setif, Algeria (1999).

[7] W. Han, M. Sofonea \& J. M. Viaño, Variational and numerical analysis of a frictionless contact problem for elastic-viscoplastic materials with internal state variables. Quarterly Journal of Mathematics and Applied Mathematics 54, 501-522 (2001).

[8] W. Han \& M. Sofonea, Quasistatic Contact Problem in Viscoelasticity and viscoplasticity. December 14 (2000).

[9] D. Klarbring, A. Mikelic \& Shillor, Frictional contact problems with normal compliance. Int. J. Engng. Sci. 26 (1988), 811-832. 
[10] N. Lebri, S. Djabi \& S. Boutechbek, Bilaleral contact with Tresca's Friction law and with internal state variables. Appied Mathematical Sciences, Vol. 2 (2008), no. 10, 479-488.

[11] N. Lebri \& S. Djabi, Frictional bilateral contact in viscoplasticity. Far East J. Math. Sci. (FJMS) 29 (2), 467-479 (2008).

[12] D. Motreanu \& M. Sofonea, Quasivariational inequalities and applications in friction contact problem with normal compliance, Advance in Mathematical Sciences and Applications (2000).

[13] M. Rochdi, Analyse variationnelle de quelques problèmes aux limites en viscoplasticité. Thèse de Doctorat d'Etat, Université de Perpignan (1997).

[14] M. Sofonea, Problèmes non-linéaires dans la théorie de l'élasticioté. Cours de Magister en Mathématiques Appliquées, Université F. Abbes, Sétif, Algérie (1993).

[15] M. Sofonea \& J. M. Viaño, Africtionless contact problem for elasticviscoplastic materials with normal compliance. Numerische Mathematik 90, 689-919 (2002).

[16] B. Teniou, Etude fonctionnelle des problèmes élasto-visco-plastiques. Ph.D. Thesis, University of Constantine, Algeria, (2000). 
